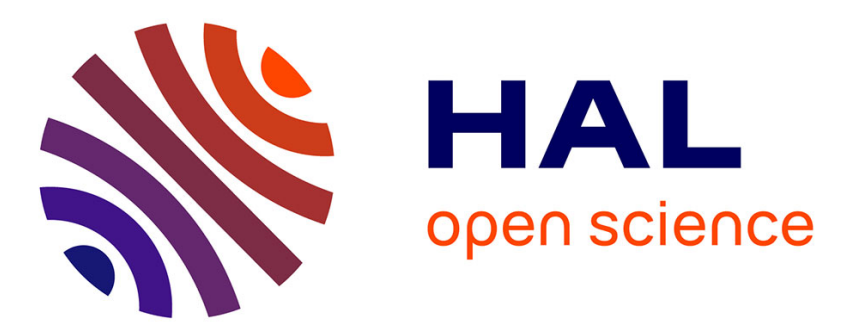

\title{
Multiphoton dissociation of macromolecular ions at the single-molecule level
}

Rodolphe Antoine, Tristan Doussineau, Philippe Dugourd, Florent Calvo

\section{To cite this version:}

Rodolphe Antoine, Tristan Doussineau, Philippe Dugourd, Florent Calvo. Multiphoton dissociation of macromolecular ions at the single-molecule level. Physical Review A : Atomic, molecular, and optical physics [1990-2015], 2013, 87 (1), pp.013435. 10.1103/PhysRevA.87.013435 . hal-02979577

\section{HAL Id: hal-02979577 \\ https://hal.science/hal-02979577}

Submitted on 11 Feb 2021

HAL is a multi-disciplinary open access archive for the deposit and dissemination of scientific research documents, whether they are published or not. The documents may come from teaching and research institutions in France or abroad, or from public or private research centers.
L'archive ouverte pluridisciplinaire HAL, est destinée au dépôt et à la diffusion de documents scientifiques de niveau recherche, publiés ou non, émanant des établissements d'enseignement et de recherche français ou étrangers, des laboratoires publics ou privés. 


\title{
Multiphoton dissociation of macromolecular ions at the single-molecule level
}

\author{
Rodolphe Antoine, ${ }^{*}$ Tristan Doussineau, Philippe Dugourd, and Florent Calvo \\ Institut Lumière Matière, UMR5306, Université Lyon 1-CNRS, Université de Lyon, 69622 Villeurbanne Cedex, France
}

(Received 26 October 2012; revised manuscript received 9 January 2013; published 31 January 2013)

\begin{abstract}
The laser-induced decay of ethylene oxide polymer ions of megadalton size has been studied in the multiphoton IR excitation regime, with fragmentation products of individual ions being monitored over long times by a trapping device. The experiment reveals several fragmentation pathways having distinct signatures at the single-molecule level that would not be accessible from studies based on statistically averaged reaction rates only. The observations are supported by dedicated molecular simulations based on a coarse-grained model, which further highlight the role played by continuous heating in such out-of-equilibrium conditions. In particular, both experiment and modeling indicate that the dissociation kinetics depends nonlinearly on heating rate.
\end{abstract}

DOI: 10.1103/PhysRevA.87.013435

PACS number(s): 33.80.Gj, 33.80.Wz, 37.10.Pq, 36.40.Qv

\section{INTRODUCTION}

The stability and fragmentation of finite systems driven by long-range forces, e.g., nuclei, clusters and nanostructures, proteins, droplets, and optical molasses, etc. [1,2], raise some fundamental issues regarding the causes and mechanisms of dissociation. How is the multidimensional energy landscape explored, and which features guide the shape evolution of the system into its fragmentation? What are the fragmentation channels, and under what conditions are they realized? An accurate description at the molecular level can be achieved when such reactions take place in the gas phase, after isolated species have interacted with neutral or ionic molecules, photons, electrons, or surfaces. Ion dissociation or transformation into different ion types can be achieved [3], with reaction products being then prone to accurate characterization by mass spectrometry [4]. Temporal information on fragmentation can also be obtained using ion storage devices [5,6]. For instance, very recently, Rahinov et al. used an image charge monitoring for lifetime measurements in an electrostatic ion beam trap [7]. However, for large molecular ions decaying into smaller fragments through a series of complex dissociation steps, mass spectrometers fail to identify the individual steps of the dissociation chains, mainly because such experiments measure intensities involving large ensembles of ions. This problem could be avoided if only a single ion were present in the experiment before being activated and if its composition could be subsequently monitored. In addition, information gained at the single-molecule level is much richer than the mere averages, as it notably provides greater insight into the physical mechanisms through the correlation between various observables.

Recent years have seen major progress in the characterization and manipulation of individual molecules. The combination of single-molecule optics [8,9] and micromanipulation [10] has enabled physical and biological systems to be studied from molecular to mesoscopic scales. For instance, the shape oscillations, stability, and fragmentation patterns of charged micrometer-size individual droplets close to the Rayleigh limit were investigated by Duft et al. [11,12] using an electrodynamic levitator. For molecular ions, the sequential breakage

\footnotetext{
*Corresponding author: rodolphe.antoine@univ-lyon1.fr
}

of positively charged aniline ions into lighter fragments at the single-molecule level was demonstrated in an ion trap [13]. Using an electrostatic trap coupled to a $\mathrm{CO}_{2}$ laser, the boundary of photofragmentation experiments using infrared (IR) multiphoton dissociation on single macroions has been pushed to the megadalton range $[14,15]$.

One difficulty of using IR multiphoton dissociation is that the concept of temperature becomes unclear, hampering simple thermodynamical analyses. This issue was addressed by Dunbar [16,17] and by Paech et al. [18], who interpreted slow heating dissociation experiments on collections of ions using phenomenological models based on master equations and Monte Carlo simulations, and obtained simple relationships between the relative laser power experienced by the ions and their temperature $[18,19]$. However, such approaches do not bring insight into the individual behaviors and ignore the possible correlation between various observables. The present work provides a combined experimental and theoretical evaluation of the fragmentation mechanisms and kinetics in the case of highly charged macromolecular ions exposed to continuous, low-energy IR photon irradiation and at the single-molecule level. For this purpose, in addition to measurements obtained on multiphoton dissociation of isolated single polymer macroions, a coarse-grained simulation model of the fragmentation dynamics of the polymer chains has been implemented to assist the interpretation of such experiments under strong out-of-equilibrium conditions.

\section{COARSE-GRAINED SIMULATION MODEL OF THE FRAGMENTATION DYNAMICS OF THE POLYMER CHAINS}

To assist the interpretation of experimental results described below, we have developed a computational model allowing explicit simulations of the time-dependent fragmentation process on a statistical footing. The model is kept as simple as possible, retaining the key physical ingredients at play in the experiment. In this coarse-grained approach the polymer is described by a set of $M$ linearly connected monomers, with the interaction between two successive monomers being taken as a Morse potential with equilibrium distance $\sigma$ and well depth $D$. The bundle shape of the polymer at equilibrium due to noncovalent forces is accounted for by adding a Lennard-Jones potential between pairs of nonadjacent monomers, with an 
identical equilibrium distance $\sigma$ and a well depth $\varepsilon$ (differing from $D$ ). Molecular dynamics simulations were performed, starting from a low-energy collapsed conformation and heating the polymer with a fixed rate $K_{h}$. To mimic IR absorption, heating is local and acts on a single bond between two successive monomers, with their relative velocity along the bond being increased by a fixed amount corresponding to a change of $\Delta E^{+}$in kinetic energy. Over the course of the trajectory, this energy is subsequently transferred to the rest of the polymer through the anharmonic couplings provided by intramolecular and noncovalent forces. In addition to this local heating process, radiative cooling is phenomenologically incorporated assuming blackbody emission. All velocities are scaled by an amount equivalent to removing a fixed amount of kinetic energy $\Delta E^{-}$to the system at a rate $K_{c}$ that varies proportionally to $T^{4}$, with $T$ being the current (shorttime averaged) temperature. In the simulations, fragmentation events are monitored by finding the distance between two successive monomers to exceed $10 \sigma$. The onset is defined as the time $t_{o}$ of the first such event. The simulation proceeds until the largest fragment is no larger than one third of the parent, thus marking the disappearance time $t_{d}$. For each heating rate $K_{h}, 10^{4}$ independent trajectories were carried out, and the results were statistically analyzed in terms of averages, fluctuations, and correlations. The computational model uses reduced units for the distance (in units of the original binding distance $\sigma=1$ ), the monomer mass (taken as unity), and energy (setting $D=2$ and $\varepsilon=0.1$ ) as well as Boltzmann's constant $k_{B}$ in such a way that energies and temperatures share the same unit. Such reduced units lead to a convenient time step of 0.01 unit for the simulations. The heating rate $K_{h}=\Delta E^{+} / t_{h}$ was varied by fixing $\Delta E^{+}=1$ and adjusting the time interval $t_{h}$ between two successive heatings. The cooling rate $K_{c}=a T^{4}=\Delta E^{-} / t_{c}$ was evaluated on the fly from the short-time averaged kinetic temperature $T$, noting that the stationary temperature under the fragmentation regime [20,21] is close to $T=0.2$ for the present polymers. Fixing $\Delta E^{-}=1$, the time delay $t_{c}$ between cooling events was adjusted in order that $K_{c}(T=0.2)=1 / 10$, which equivalently sets the constant $a$ to 62.5 reduced units.

\section{EXPERIMENT}

Experiments were performed on an electrostatic ion trap coupled to an electrospray ionization source and a continuous $\mathrm{CO}_{2}$ laser, as described recently [15]. We used polyethylene oxide (PEO) ions with a nominal average molecular weight of $7 \mathrm{MDa}$ (Sigma-Aldrich). The mass distribution for these ions recorded by charge-detection mass spectrometry [22,23] exhibits a maximum at 6.2 MDa, as well as a high-mass tail caused by the dispersion in the degree of polymerization for such macromolecules, which extends up to $~ 30$ MDa [24]. Solutions were prepared in water/acetonitrile (50:50 volume ratio) at a concentration of $0.02 \mathrm{~g} / \mathrm{L}$ and electrosprayed in positive mode. Gas-phase ions were transmitted through an ionic train containing a hexapole ion guide and ionic lenses, and directed toward two charge detection devices (CDD). The first MS stage combined with an ion gate allows $\mathrm{m} / \mathrm{z}$ and charge selection of a specific megadalton ion of interest before injection into the trap. The trapped ions had an initial mass and charge of $20 \pm 5 \mathrm{MDa}$ and $1250 \pm 300 e$, respectively. When the selected ion enters the second CDD device, it triggers a circuit that places the potentials on the entrance and exit electrodes (ion mirrors) to predetermined values chosen to reflect ions in certain ranges of $z$ and $m / z$. With the current setup, the noise level in the pickup signal is $\sim 400 e$, and fragment ions with total charges lower than $400 e(\sim 30 \%$ of initial total charge) cannot be detected. During the trapping time, the ions can be fragmented by exposure to the $\mathrm{CO}_{2}$ laser (cw laser at $\lambda=10.6 \mu \mathrm{m}$, output power of $25 \mathrm{~W}$, diameter of $8.1 \mathrm{~mm}$ in the trap). The laser beam is reflected by two gold-coated copper mirrors, after which it is injected on the axis of the ion trap through a $\mathrm{ZnSe}$ window fitted on the rear of the CDD chamber. For laser power dependency experiments, the laser output was chopped electronically with a $5-\mathrm{kHz}$ pulse train chosen to realize duty cycles between $10 \%$ and $99 \%$ [15].

\section{ANALYSIS OF RAW TIME DOMAIN SIGNAL FOR A SINGLE TRAPPED ION}

In the ion trap, single trapped ions undergo several hundred round trips between the ion mirrors. The corresponding image current generated by the round trips of ions in the trap (oscillations) is then recorded as a function of time. Figure 1 shows the experimental raw time domain signal [15] for a trapped ion created by a single highly charged electrospray ion of PEO (mass of $20 \mathrm{MDa}$ and 1550 charges) travelling back and forth through the ion trap. The Fourier transform of this signal (right panel in Fig. 1) shows a peak at $13.8 \mathrm{kHz}$, indicating the fundamental oscillation frequency, which corresponds to an $m / z$ of $12900 \mathrm{Da} / e$. The first harmonic can be seen at $27.6 \mathrm{kHz}$. This single ion was trapped for $\sim 52 \mathrm{~ms}$, during which time it cycled $\sim 750$ times through the detector tube. The shape and amplitude of the raw signal are roughly the same, which means that no significant charge loss is observed during the trapping time. As an illustration, Fig. 1 displays the evolution of the total charge of this trapped ion as function of the time for each round trip.

As the laser is switched on during the ion trapping time, drastic changes are observed both in the trapping duration and the shape of the oscillations. As shown in Fig. 2, for selected raw time domain signals for three different trapped ions, under continuous irradiation by the $\mathrm{CO}_{2}$ laser $\left(14.6 \mathrm{~W} / \mathrm{cm}^{2}\right)$, the ion has a trapping time that does not exceed $20 \mathrm{~ms}$. In Fig. 2(a), the charge does not change significantly during the first $10 \mathrm{~ms}$. Then its amplitude drops, and for trapping times longer than $16 \mathrm{~ms}$ the particle is no longer detected. During the last stages, the total charge gradually decreases before the ion is lost or cannot be detected, a type of raw time domain signal referred to as "funnel." The regular charge loss as a function of time observed for PEO ions shows that small units losses are favored.

However, markedly different signals are also recorded, as illustrated by the traces displayed in Figs. 2(a) and 2(b). These traces correspond to "staircase" types of raw time domain signals. For the trace in Fig. 2(b), the precursor ion suddenly loses a large amount of charge $(\sim 35 \%$ of initial charge) after $\sim 8.5 \mathrm{~ms}$, and then the amplitude of the charge remains almost constant for $\sim 8 \mathrm{~ms}$ before the ion can no longer be detected. Interestingly, in some rare cases, raw time domain signals display multiple "staircase" fragmentations. 

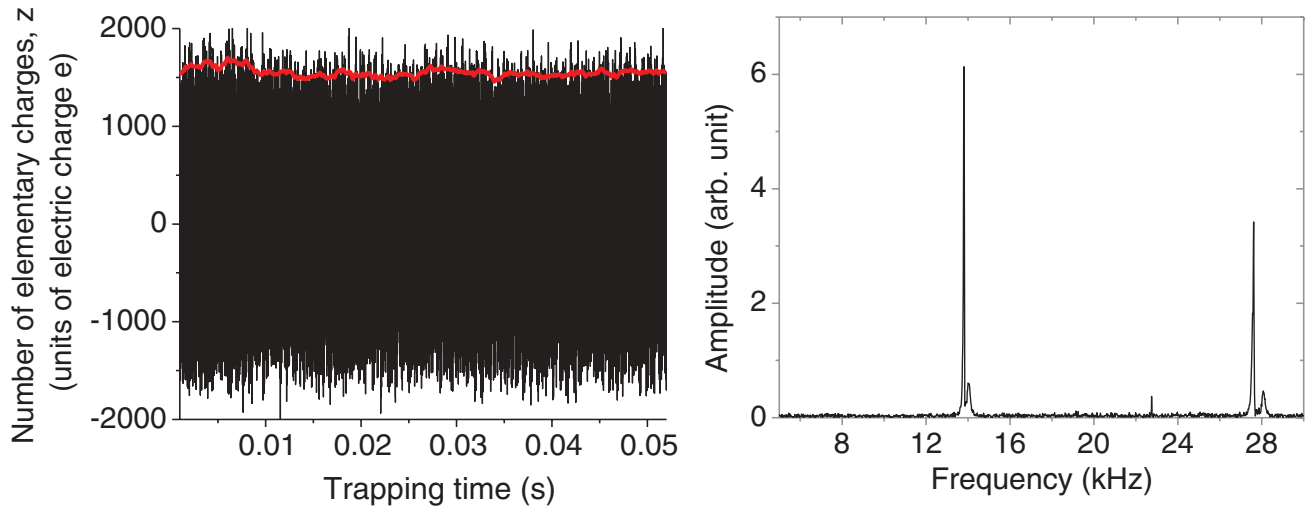

FIG. 1. (Color online) (left) Experimental raw time domain signal for a trapped PEO ion (mass of 20 MDa and 1550 charges). The signal shown here was passed through a $60-\mathrm{kHz}$ FFT low-pass filter. The red curve shows the evolution of the total number of elementary charges of trapped ions as a function of time. A smoothing of raw data using adjacent averaging (ten points) has been used. (right) The Fourier transform of the raw signal with a fundamental oscillation frequency of $13.8 \mathrm{kHz}$ and a first harmonic at $27.6 \mathrm{kHz}$.

The trace in Fig. 2(c) shows such an example, where after $\sim 11 \mathrm{~ms}$ the precursor ion suddenly loses $\sim 15 \%$ of total charge. Then the amplitude of the charge remains almost constant for $\sim 5.5 \mathrm{~ms}$, and finally, the first fragment ion dissociates, leading to another fragment that has lost $\sim 35 \%$ of the first fragment ion charge. This second fragment is stable for $3.5 \mathrm{~ms}$, and then a new dissociation occurs. The resulting third fragment further decays and is no longer detectable $\sim 23 \mathrm{~ms}$ after the onset time.

It is important to emphasize here that the present ion trap device can only monitor the evolution of the total charge of trapped ions as a function of time. The mass of the photofragments can be estimated indirectly assuming that the mass-to-charge ratio remains constant during fragmentation; i.e., the charges are homogeneously distributed in space along the polymer and remain so after fragmentation. The fundamental frequency $f$ of the oscillatory motion extracted from fast Fourier transform (FFT) (as shown in Fig. 1) can be used to determine the $m / z$ ratio through the simple relation [25]

$$
f=\sqrt{\frac{C}{2 E_{0} m / z}}
$$

where $C$ is a constant that depends on the geometry of the ion trap and the voltages applied on ion mirrors. $E_{0}$ is the kinetic energy of the trapped ion. This formula can be used to extract $\mathrm{m} / \mathrm{z}$ from in-trap ions since $f, C$, and $E_{0}$ can be known precisely $[25,26]$. However, when photofragmentation occurs, both the $\mathrm{m} / \mathrm{z}$ ratio and the kinetic energy $E_{0}$ of the photofragments can be different from the parent ion, and then accurate measurements of $\mathrm{m} / \mathrm{z}$ for photofragments are no longer possible. The Fourier transforms of the experimental raw time domain signals for parent (between 0 and $8 \mathrm{~ms}$ ) and fragment (between 8.7 and $16 \mathrm{~ms}$ ) ions lead to fundamental frequencies of 15.1 and $13.4 \mathrm{kHz}$, respectively. In general, for this type of raw time domain signal, frequencies for fragments are different than those measured for the parent ion.

In fact, the criteria for trapping stability in such electrostatic ion traps are nontrivial and must satisfy both spatial and temporal focusing conditions [27]. Trajectory simulations using SIMION 7.0 [28] indicate that an ion with a mass of
$20 \mathrm{MDa}$ and 1250 charges can be trapped in a specific range of initial velocities (from 310 to $1400 \mathrm{~m} / \mathrm{s}$ ) by the potential well. However, these simulations show that the trapping capability is strongly sensitive to the entrance angle and the ion position of the incoming ion [15,29].

To better address this diversity of fragmentation processes for PEO ions at the single-molecule level, the ratio $z_{F} / z_{P}$ between the total charge of the largest fragment $z_{F}$ and the total charge of the precursor ion $z_{P}$ was measured for each transient ion as a function of time after the onset time of fragmentation. The evolution of the ratio $z_{F} / z_{P}$ recorded for 85 individual ions stored in the electrostatic ion trap, under continuous irradiation of the $\mathrm{CO}_{2}$ laser $\left(14.6 \mathrm{~W} / \mathrm{cm}^{2}\right)$, is shown in Fig. 3 as a function of time after onset. The three fragmentation patterns of the traces in Figs. 2(a), 2(b), and 2(c) correspond to clearly distinct pathways that would not be resolved from the average signal. It appears that, under such continuous laser irradiation, $~ 57 \%$ of the traces display a sudden loss of ions (without trapping any fragment ions); $\sim 20 \%$ are of the "funnel" type, $\sim 20 \%$ are of the "single-staircase" type, and the remaining $3 \%$ are of the "multiple-staircase" type. These proportions do not significantly depend on the laser power. The individual traces provide the opportunity to extract correlated data, such as the onset and dissociation times, as discussed below.

\section{CORRELATION BETWEEN ONSET AND DISSOCIATION TIMES IN MULTIPHOTON DISSOCIATION OF MACROMOLECULAR IONS}

The delay $\delta t=t_{d}-t_{o}$ between onset and disappearance marks the time needed for the polymer to break into sufficiently small pieces once a bond has broken, as a result of subsequent heating. This quantity can be evaluated independently for each event or trajectory, thereby carrying more information than statistical averages alone. $\delta t$ was estimated in both the experiment and the simulation and is shown in Fig. 4 against the onset time as a two-dimensional density plot. There is some general anticorrelation between $\delta t$ and $t_{o}$, especially in the simulation, suggesting that trajectories dissociating earlier need a longer time to produce small fragments. The 

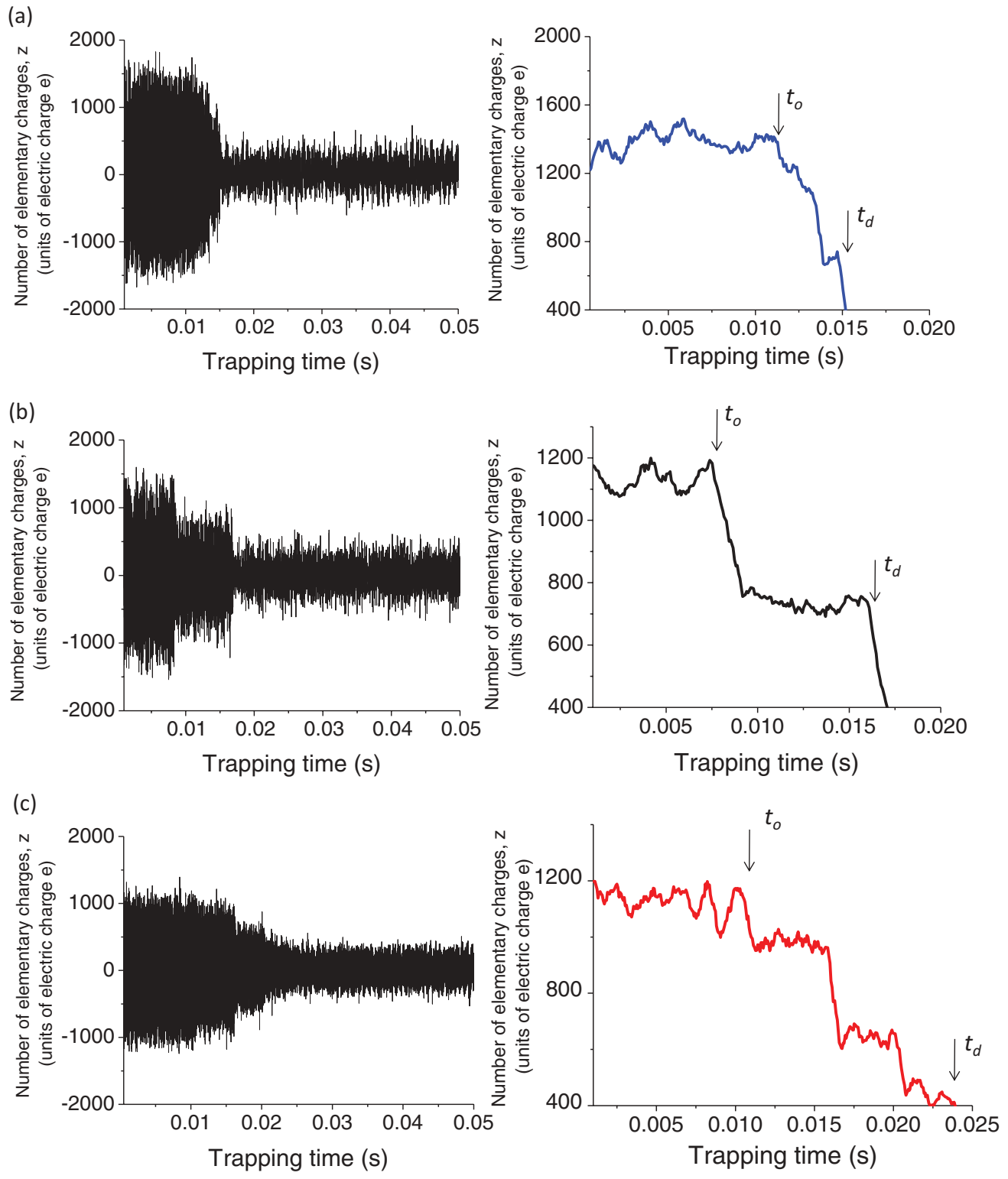

FIG. 2. (Color online) (left) Experimental raw time domain signal for three trapped $\mathrm{PEO}$ ions under continuous irradiation of the $\mathrm{CO}_{2}$ laser $\left(14.6 \mathrm{~W} / \mathrm{cm}^{2}\right)$. The signals were passed through a $60-\mathrm{kHz}$ FFT low-pass filter. (right) Evolution of the total number of elementary charges of trapped ions as a function of time. A smoothing of raw data using adjacent averaging (ten points) has been used. The onset and disappearance times $\left(t_{o}\right.$ and $t_{d}$ ) for each trace are shown as arrows.

corresponding experimental data, however, show a significant population at low delay and low onset times in a region that is excluded by the simulation. This behavior may be due to some dispersion in the experimental initial conditions in both charge and mass of the macroions and also to nonstatistical heating of selected ions in the experiment, whereas a single size and a constant heating rate were considered in the simulation. The anticorrelation feature, also present in the experiment, would seem counterintuitive if we assumed the polymers resulting from the first dissociation to share the same temperature. Thus, the longer delay to disappearance implied by the anticorrelation shows that such polymers were initially colder when the first fragmentation occurred and that a roughly constant exposure time is required.

\section{DISSOCIATION KINETICS OF MACROMOLECULAR IONS}

Interpreting the observed kinetics for the dissociation of PEO ions requires monitoring a large set of traces of individual ions to construct a density distribution of dissociation rates for a collection of ions. For each laser power, $\sim 100$ traces of individual ions were analyzed, and the normalized ion count at a given time (ranging from 1 to $20 \mathrm{~ms}$ ) was obtained by the number of traces for which the ion was still detectable at this time, further normalized by the initial number of traces considered. The inset in Fig. 5 shows the relative abundance of MDa PEO ions in log scale as a function of time and for different laser powers. During an initial time period, a small decay is observed. This onset time of fragmentation decreases with laser intensity and is required to heat the ions from room 


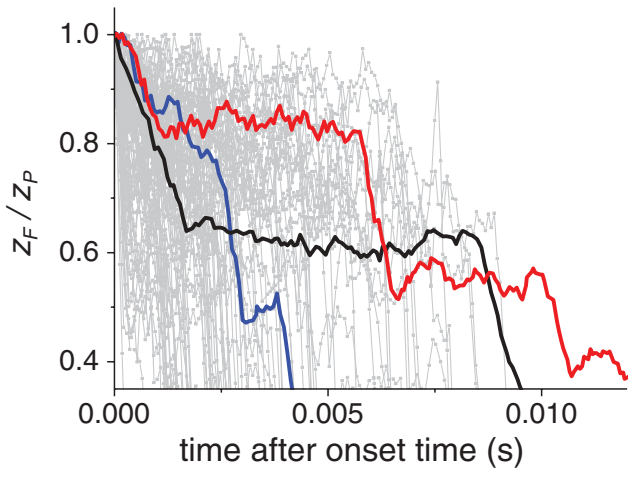

FIG. 3. (Color online) Evolution of the ratio $z_{F} / z_{P}$ between the total charge of the largest fragment $z_{F}$ and the total charge of the precursor ion $z_{P}$ recorded for 85 individual ions stored in the electrostatic ion trap under continuous irradiation of the $\mathrm{CO}_{2}$ laser (14.6 $\mathrm{W} / \mathrm{cm}^{2}$ ) as a function of time after onset. The evolutions of the total charge of the three trapped ions displayed in Fig. 2 are highlighted.

temperature until they are hot enough for dissociation to start. After the onset time, ion dissociation produces an exponential decrease in parent ion count (straight lines in the inset in Fig. 5). Here the kinetics is measured after the onset time only. The rate of dissociation increases with laser intensity (see Fig. 5) and can be evaluated for each laser power from the linear fit using $k_{\mathrm{diss}}=-\frac{d \ln \left(C_{I}\right)}{d t}$, where $C_{I}$ is the ion count. Figure 5 displays the evolution of $k_{\text {diss }}$ as a function of laser intensity in an Arrhenius-like plot. The plot of $k_{\text {diss }}$ versus
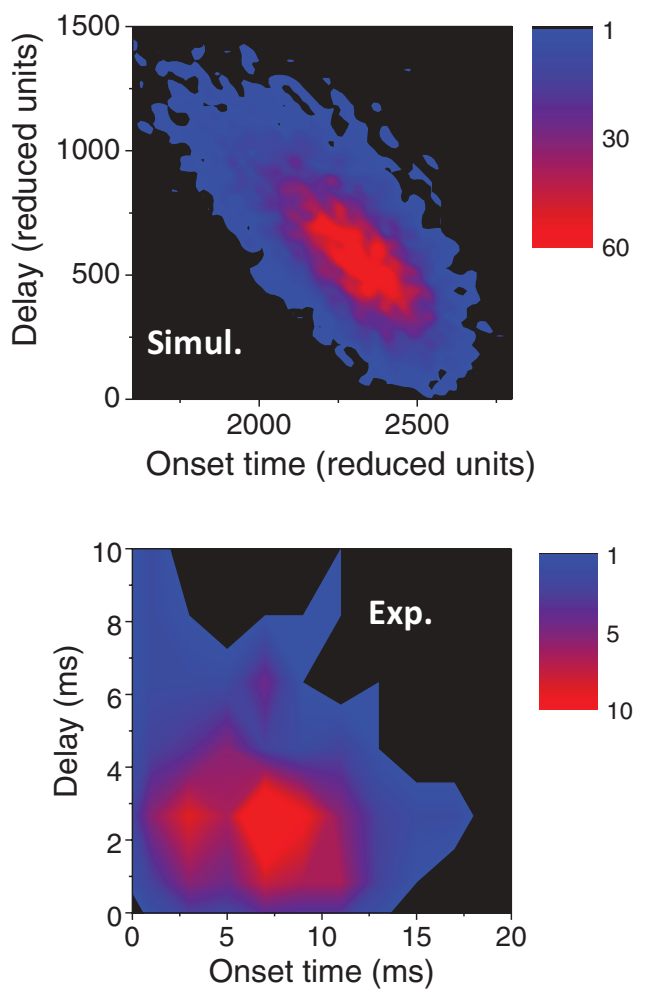

FIG. 4. (Color online) Distribution of time delay $\delta t=t_{d}-t_{0}$ between the onset time of fragmentation and the disappearance time vs the onset time $t_{o}$. (top) Simulation results obtained for $M=400$ and a heating rate of $K_{h}=10$ units. (bottom) Experimental data for laser power of $14.6 \mathrm{~W} / \mathrm{cm}^{2}$.

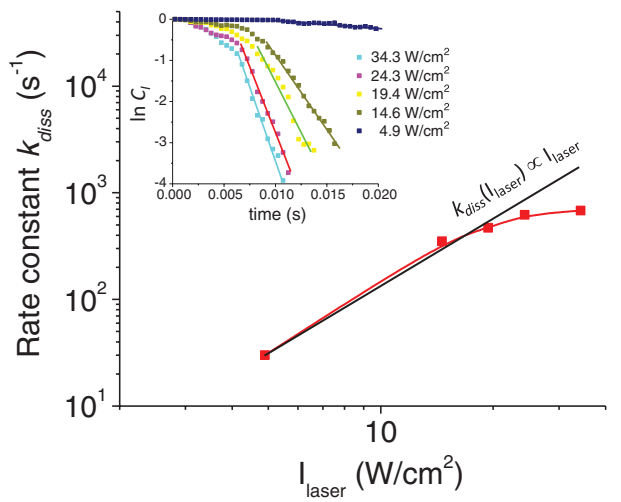

FIG. 5. (Color online) Logarithmic plot of the unimolecular dissociation rate constant $k_{\text {diss }}$ vs laser intensity for PEO ions. Smoothed polynomial and linear curves are given to guide the eyes (solid lines). The inset shows the ion count vs time for the dissociation of PEO ions under different $\mathrm{CO}_{2}$ laser powers, in semilogarithmic scale. The lines highlight the regimes where the rate constants were extracted after the onset of fragmentation.

laser intensity in logarithmic scale reveals a concave curvature. At high laser power, the onset times of fragmentation are short (typically a few milliseconds), and it is possible that dissociation of excited ions competes with photon absorption or emission, with ion dissociation no longer in the so-called rapid exchange limit (REX) [30], for which the internal energy distribution of excited ions follows a Boltzmann distribution at any time. As the laser power increases, the dissociation rate increases as well, and ions with slower radiative exchange rates fall outside the REX limit, while those exchanging faster remain in the REX limit until slightly higher laser powers are used. If dissociation is fast relative to activation, the thermal distribution is depleted, and $k_{\text {diss }}$ varies more slowly against high laser intensities [31].

In contrast with the phenomenological adjustments used to extract the experimental decay rates over long times from the assumption of a fixed temperature, the fragmentation kinetics can be characterized with this model (introduced in Sec. II) over the entire time range, taking into account the continuous heating experienced by the polymer. Because of the simultaneously ongoing heating, the probability $P(t)$ at time $t$ does not follow a simple exponential decay. It is necessary to account for the variations of the effective temperature with time, which we write as $T(t)=T(0)+\gamma t$. Assuming the fragmentation still obeys first-order kinetics, $P(t)$ satisfies $d P / d t=-k(t) P(t)$, with $k(t)=k(0) \exp [-D / T(t)]$, leading to

$$
P(t)=\exp \left[-k_{0} \int_{0}^{t} \exp \left(-\frac{D}{T(0)+\gamma t^{\prime}}\right) d t^{\prime}\right] .
$$

Fixing $D=2$ and $T(0)=0.01$ from the model inputs, $k_{0}$ and $\gamma$ are fitted to reproduce the variations of $P(t)$ in the simulations. The equation above accurately represents the simulated variations of $P(t)$, as shown in the inset in Fig. 6 for two values of the heating rate. Defining the effective fragmentation rate as the value of $k(t)$ when $P(t)$ equals one half, we find the variation of this effective fragmentation rate as a function of the heating rate $K_{h}$. Those variations, given in Fig. 6, show some essentially linear behavior, with some attenuation at high heating rates that is consistent with the observed saturation in 


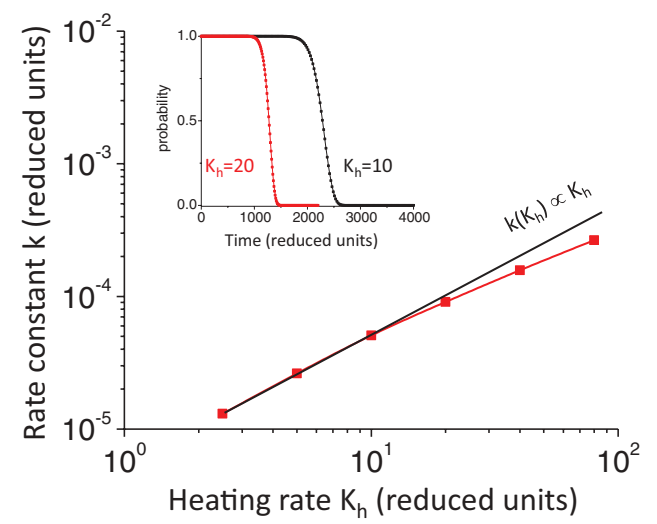

FIG. 6. (Color online) Fragmentation rate constant defined at the time where the 400 -bead polymer drops below $50 \%$ probability to remain intact as a function of the heating rate $K_{h}$. The inset shows the time variations of this probability for two values of $K_{h}$ (squares) and their representation using Eq. (2) (solid lines).

our experiment (as seen in Fig. 5). This attenuation conveys the particularly out-of-equilibrium character of dissociation under strong heating, where temperature itself is not well defined.

The statistical analysis of individual trajectories brings further insight into the out-of equilibrium fragmentation process, as a complement to the experimental signatures. There is, for instance, only a minor preference for the outer bonds of the polymer to break relative to the central bonds, and the general shape of the polymer as it dissociates is largely unfolded. This random behavior of fragmentation is due to the homogeneity of the chemical bonding in such polymer chains for which the total charge remains moderate.

\section{CONCLUSION}

In summary, the effect of IR multiphoton absorption on heating and dissociation times for a large ensemble of ions of polymer chains in the megadalton size-range was investigated experimentally and computationally. While still at the molecular scale, the measurements were achieved on single macroions stored for dozens of milliseconds. To assist the interpretation of experimental results, we have developed a computational model allowing explicit simulations of the time-dependent fragmentation process on a statistical footing. This joint approach would be valuable for other systems presenting chemical heterogeneity, such as supramolecular complexes with covalent and noncovalent bonds, including DNA multiplexes or protein assemblies. A study at the single-ion level would allow fragmentation scenarios to be accurately detailed, opening the way to a more complete understanding of the key parameters that drive covalent and noncovalent bond dissociation. Another relevant situation concerns highly charged systems close to the Rayleigh limit, such as submicrometer-size droplets or products of electrospray ionization sources, and for which multiple dissociation channels (evaporation, fission, etc.) compete with each other.

\section{ACKNOWLEDGMENTS}

We are grateful to the ANR for financial support of this work (Grants No. ANR-08 BLAN-0110-01 and No. ANR11-PDOC-032-01). The authors would like to thank Xavier Dagany, Christian Clavier, Michel Kerleroux, Marc Barbaire, Jacques Maurelli, and Congyu Bao for their invaluable assistance.
[1] J. Jortner, I. Last, and Y. Levy, Int. J. Mass Spectrom. 249-250, 184 (2006).

[2] I. Last, Y. Levy, and J. Jortner, Proc. Natl. Acad. Sci. USA 99, 9107 (2002).

[3] Fundamentals of Gas Phase Ion Chemistry, edited by K. R. Jennings (Kluwer Academic, Dordrecht, Boston, 1991).

[4] S. A. McLuckey and J. M. Wells, Chem. Rev. 101, 571 (2001).

[5] J. U. Andersen, P. Hvelplund, S. Brøndsted Nielsen, S. Tomita, H. Wahlgreen, S. P. Møller, U. V. Pedersen, J. S. Forster, and T. J. D. Jørgensen, Rev. Sci. Instrum. 73, 1284 (2002).

[6] J. Laskin and J. H. Futrell, Mass Spectrom. Rev. 24, 135 (2005).

[7] I. Rahinov, Y. Toker, O. Heber, D. Strasser, M. Rappaport, D. Schwalm, and D. Zajfman, Rev. Sci. Instrum. 83, 033302 (2012).

[8] A. D. Mehta, M. Rief, J. A. Spudich, D. A. Smith, and R. M. Simmons, Science 283, 1689 (1999).

[9] S. Weiss, Science 283, 1676 (1999).

[10] O. A. Saleh, J.-F. Allemand, V. Croquette, and D. Bensimon, Chem. Phys. Chem. 6, 813 (2005).

[11] D. Duft, T. Achtzehn, R. Müller, B. A. Huber, and T. Leisner, Nature (London) 421, 128 (2003).

[12] D. Duft, H. Lebius, B. A. Huber, C. Guet, and T. Leisner, Phys. Rev. Lett. 89, 084503 (2002).
[13] K. Højbjerre, D. Offenberg, C. Z. Bisgaard, H. Stapelfeldt, P. F. Staanum, A. Mortensen, and M. Drewsen, Phys. Rev. A 77, 030702(R) (2008).

[14] T. Doussineau, R. Antoine, M. Santacreu, and P. Dugourd, J. Phys. Chem. Lett. 3, 2141 (2012).

[15] T. Doussineau, C. Y. Bao, C. Clavier, X. Dagany, M. Kerleroux, R. Antoine, and P. Dugourd, Rev. Sci. Instrum. 82, 084104 (2011).

[16] R. C. Dunbar, J. Chem. Phys. 95, 2537 (1991).

[17] R. C. Dunbar, Mass Spectrom. Rev. 11, 309 (1992).

[18] K. Paech, R. A. Jockusch, and E. R. Williams, J. Phys. Chem. A 106, 9761 (2002).

[19] M. Schäfer, C. Schmuck, M. Heil, H. J. Cooper, C. L. Hendrickson, M. J. Chalmers, and A. G. Marshall, J. Am. Soc. Mass Spectrom. 14, 1282 (2003).

[20] F. Calvo, Phys. Rev. A 71, 041201(R) (2005).

[21] M. Schmidt, T. Hippler, J. Donges, W. Kronmüller, B. von Issendorff, H. Haberland, and P. Labastie, Phys. Rev. Lett. 87, 203402 (2001).

[22] S. D. Fuerstenau and W. H. Benner, Rapid Commun. Mass Spectrom. 9, 1528 (1995).

[23] J. W. Smith, E. E. Siegel, J. T. Maze, and M. F. Jarrold, Anal. Chem. 83, 850 (2011). 
[24] T. Doussineau, M. Kerleroux, X. Dagany, C. Clavier, M. Barbaire, J. Maurelli, R. Antoine, and P. Dugourd, Rapid Commun. Mass Spectrom. 25, 617 (2011).

[25] N. C. Contino and M. F. Jarrold, Int. J. Mass Spectrom. (2013), doi: 10.1016/j.ijms.2012.07.010.

[26] N. Contino, E. Pierson, D. Keifer, and M. Jarrold, J. Am. Soc. Mass Spectrom. 24, 101 (2013).

[27] A. L. Rockwood, J. Am. Soc. Mass Spectrom. 10, 241 (1999).
[28] D. A. Dahl, SIMION 7.0 3D, version 7.0 (2004), Idaho National Engineering Laboratory, Idaho Falls.

[29] S. Merenbloom, D. Mortensen, and E. R. Williams in Proceedings of the 60th American Society of Mass Spectrometry Conference on Mass Spectrometry and Allied Topics [J. Am. Soc. Mass Spectrom. 23(1), Supplement, 34 (2012)].

[30] R. C. Dunbar and T. B. McMahon, Science 279, 194 (1998).

[31] R. A. Jockusch, K. Paech, and E. R. Williams, J. Phys. Chem. A 104, 3188 (2000). 